the clinical and structural results, in particular, the levels improved after the dose was increased to $10 \mathrm{mg}$ bid.

\begin{tabular}{|c|c|c|c|c|}
\hline Patient & CDAI BSL & $\begin{array}{c}\text { CDAI } 3 \text { Mnth } \\
\text { All taking } 5 \mathrm{mg} \text { bid }\end{array}$ & $\begin{array}{l}\text { CDAI12 Mnth } \\
\text { Remain on } \\
5 \mathrm{mg} \text { bid }\end{array}$ & $\begin{array}{l}\text { CDAl } 12 \text { Mnth } \\
\text { Dose } \\
\text { Increased } \\
10 \mathrm{mg} \text { bid }\end{array}$ \\
\hline 001 & 24.9 & 0.6 & 6 & \\
\hline 002 & 56.1 & 20.9 & & 2.7 \\
\hline 003 & 45.9 & 27.4 & & 2.3 \\
\hline 004 & 65.2 & 33.8 & & 0 \\
\hline 005 & 56.1 & 15.5 & & 3.1 \\
\hline 006 & 34.7 & 38.9 & & 19.4 \\
\hline 007 & 27.9 & 56 & & 1.5 \\
\hline 008 & 34.3 & 18.4 & & 1.8 \\
\hline 009 & 43.6 & 3.3 & 2.3 & \\
\hline 010 & 33.5 & 12.6 & & ET \\
\hline 011 & 32.7 & 5.1 & ET & \\
\hline 012 & 25.3 & 13 & & 0 \\
\hline 013 & 21.2 & 2.4 & ET & \\
\hline 014 & 21.6 & 9.9 & & 2.9 \\
\hline 015 & 21.6 & 5.2 & & ET \\
\hline 016 & 27.7 & 0.4 & ET & \\
\hline 018 & 31.8 & 16.9 & & 2.1 \\
\hline 019 & 27.3 & 0.9 & & 5.2 \\
\hline 020 & 14.5 & 5.2 & & ET \\
\hline 027 & 30.5 & 2.1 & 1.9 & \\
\hline
\end{tabular}

Conclusions: Our results suggest that a significant number of patients treated with the standard dose of $5 \mathrm{mg}$ bid may potentially have improved outcomes including LDA or remission when treated at a higher dose $(10 \mathrm{mg} \mathrm{bid})$. As is evidenced by the results in this study, 11 of the 14 patients had significant improved response after treatment with the step up dose. It would appear that this improved result occurs by 3 months of therapy. Furthermore, the structural findings correlate in large part to the clinical findings showing stabilization or improvement in the majority of patients. A larger study is needed to validate these clinical and structural responses as well as to evaluate the safety outcomes using $10 \mathrm{mg}$ bid for intervals of more than 12 months.

Disclosure of Interest: None declared

DOI: 10.1136/annrheumdis-2017-eular.1806

\section{AB0262 EVALUATION OF PATIENT REPORTED OUTCOME USING RAPID3 AND HAQ-DI COMPARED TO DAS28: EXPERIENCE FROM ROUTINE CLINICAL PRACTICE IN MALAYSIA}

N. Mohd Jamid $^{1}{ }^{1}$, B. D'Souza ${ }^{1}$, H.C. Hong ${ }^{1}$, N. Mohd Noor ${ }^{1}$, C.K. Cheah ${ }^{1}$, Y.L. Lee ${ }^{2}$, S.C. Gun ${ }^{1} .{ }^{1}$ Hospital Tuanku Jaafar, Seremban; ${ }^{2}$ PFIZER, Kuala Lumpur, Malaysia

Background: Patient reported outcome (PRO) is an important measure to physician in management of patient with rheumatic diseases. Health assessment questionnaire disability index (HAQ-DI) is the most widely used $\mathrm{PRO}$ tool in rheumatoid arthritis (RA) clinical trials. Previous studies have shown that HAQDI correlates well with disease activity score of 28-joints (DAS28). However, routine assessment of patient index data 3 (RAPID3) is much simpler and faster questionnaire to score.

Objectives: This study aims to evaluate the correlation between RAPID3 and DAS28 compared to HAQ-DI and DAS28 in our population.

Methods: RA patients who received routine treatment in Hospital Tuanku Ja'afar from March to November 2016 were included in this study. Validated RAPID3 and HAQ-DI questionnaire were made available in other languages; Malay, Chinese and Tamil, for patients who were not English literate. Descriptive analysis were conducted. Pearson correlation was used to measure the correlation between these PRO tools while area under the curve of the receiver operating characteristic (ROC) curves evaluate the sensitivity to detect disease activity. DAS28-ESR and DAS28-CRP were used as the reference variable in ROC analysis to stratified the disease activity into two groups; low (remission and low) and high (moderate and high) disease activity.

Results: A total of 400 patients completed PRO assessments were available for analysis. The median age of our cohort was 57 years old (range 22 to 88 ) and $87.5 \%$ were female. Ethnic distribution in this cohort were as follows; $38.5 \%$ Indian, 31.8\% Malay and 27.8\% Chinese. Both RAPID3 $(r=0.74, p<0.001)$ and HAQ-DI $(r=0.57, p<0.001)$ were correlated with DAS28-ESR. The area under the curve was significantly higher in RAPID3 (83\%) compared to HAQ-DI (75\%) which implied greater performance in discriminating low and high disease activity using DAS28-ESR as reference. We observed similar performance trend between RAPID3 (92\%) and HAQ-DI (82\%) compared to DAS28-CRP.

Conclusions: In conclusion, RAPID3 is an effective quantitative measure of disease activity compared to HAQ-DI in our population. Furthermore, RAPID3 yield similar disease activity categories as DAS28 without the need of joint counts
ROC Curve to discriminate low and high disease activity using DAS28CRP as

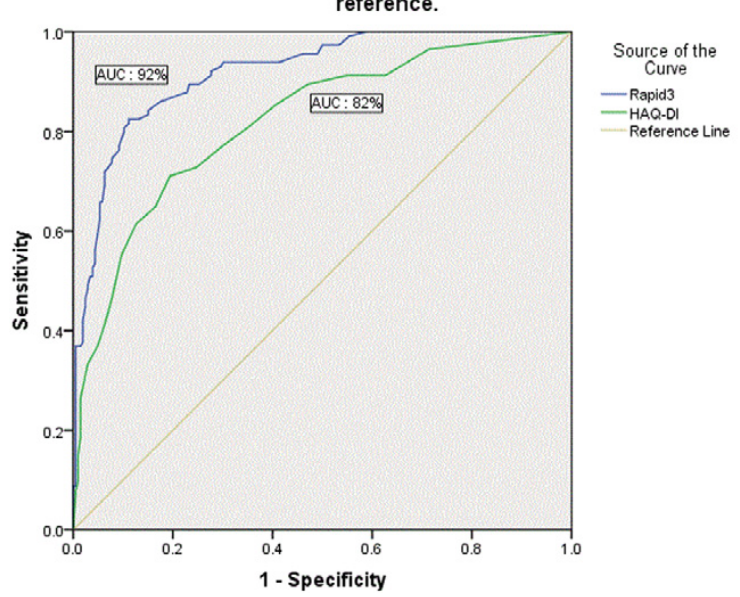

and laboratory tests. Hence is an informative assessment of disease activity in busy clinic settings.

References:

[1] Patient Reported Outcome in Rheumatoid Arthritis Clinical Trials. AnaMaria Orbai, Clifton O. Bingham III. Current Rheumatology Reports. 2015 Apr;174(4):501

[2] RAPID3 (Routine Assessment of Patient Index Data) on an MDHAQ (Multidimensional Health Assessment Questionnaire): Agreement with DAS28 (Disease Activity Index) and CDAI (Clinical Disease Activity Index) Activities Categories, Scored in Five Versus More Than Ninety Seconds. T. Pincus, C.J. Sweringen, M.J. Bergman, C.L. Colglazier, A.T. Kaell, A.M. Kunath, E.L. Siegel, Y, Yazici. Arthritis Care Res 2010;62:181

Disclosure of Interest: None declared

DOI: 10.1136/annrheumdis-2017-eular.2166

\section{AB0263 PREDICTIVE VALUE OF A SINGLE MEASUREMENT OF THE MULTI-BIOMARKER DISEASE ACTIVITY (MBDA) SCORE FOR DISEASE FLARES WITHIN 6 AND 12 MONTHS IN RHEUMATOID ARTHRITIS PATIENTS USING TUMOR NECROSIS FACTOR INHIBITORS AND CONVENTIONAL SYNTHETIC DMARDS}

P.M.T. Klooster ${ }^{1}$, M. Ghiti-Moghadam ${ }^{1}$, F. Lamers-Karnebeek ${ }^{2}$, H. Vonkeman ${ }^{3}$, E. Sasso ${ }^{4}$, P.V. Riel ${ }^{2}$, M.V.D. Laar ${ }^{3}$, T.L. Jansen ${ }^{5} .{ }^{1}$ Rheumatology, University of Twente, Enschede; ${ }^{2}$ Rheumatology, Bernhoven hospital, Uden; ${ }^{3}$ Rheumatology, Medical Spectrum Twente, Enschede, Netherlands; ${ }^{4}$ Medical\&Scientific Affairs, Crescendo Bioscience, SanFrancisco, United States; ${ }^{5}$ Rheumatology, VieCuri MC, Venlo, Netherlands

Background: Rheumatoid Arthritis (RA) patients in the maintenance phase should be treated by escalating antirheumatics up to stable low disease activity and then often are treated with tumor necrosis factor inhibitors (TNFi) and conventional synthetic disease modifyers (csDMARD). Once this phase has been reached the risk of flare is an issue once drugs are to be tapered or discontinued. Objectives: To examine the ability of the multi-biomarker disease activity (MBDA) score as predictor for disease flare in rheumatoid arthritis (RA) patients with stable low disease activity using tumor necrosis factor inhibitors (TNFi) and conventional synthetic disease modifyers (csDMARD).

Methods: Data were used from the continuation control group of the Dutch multicenter, open-label, POET trial, in which patients with stable low disease activity (disease activity score [DAS28] $<3.2$ for at least 6 months] were randomized to either stop or continue TNFi treatment. Three indicators of disease relapse were assessed: 1) flare based on DAS28 (DAS28 $>3.2$ with $\triangle$ DAS28 $>0.6$ ), 2) flare based on escalation of any DMARD therapy, and 3) flare based on physician-reported disease activity. Associations between baseline MBDA score and meeting the different criteria for disease flare within 6 or 12 months of follow-up were examined using univariate analysis and multivariate logistic regression adjusting for baseline DAS28 score.

Results: For this post-hoc analysis, baseline serum samples to measure MBDA scores were available for $225 / 286(78.7 \%)$ of the patients randomized to the TNFi continuation group (88.9\% also used methotrexate, another $8.0 \%$ used another csDMARD and $3.1 \%$ used no csDMARD): $86.2 \%$ with a first TNFi, $11.6 \%$ with second TNFi and $2.2 \%$ with a third TNFi. Within 12 months, $19.1 \%$ of patients had experienced a DAS28 flare, $12.0 \%$ had medication escalation and $8.0 \%$ had $\geq 1$ physician-reported flare. Median time to DAS28-based flare was 26 weeks (IQR:13-28). Univariately, patients with high baseline MBDA (>44) scores $(\mathrm{n}=31)$ were at increased risk of experiencing a DAS28 flare within 6 (OR $=4.39$, $\mathrm{P}=0.001)$ or $12(\mathrm{OR}=2.78, \mathrm{P}=0.015)$ months. MBDA scores were not associated with increased risk of medication escalation or physician-reported flare. After adjustment for baseline DAS28 scores, high MBDA score remained predictive for risk of flare within 6 months $(O R=3.15, P=0.017)$, but not for flare within 12 months $(\mathrm{OR}=2.05, \mathrm{P}=0.107)$ 